\title{
Fuzzification using Approximation with Fuzzy Controllers to Extract Structure and Motion Environments in Dynamic Humanoid Robotic System from the Opted and Forced Uncertainty by Quantization in Nonmonotone Neural Networks.
}

\author{
Prof. Dr. Ashok Kumar Ramadoss. PhD
}

\begin{abstract}
Fuzzy controllers are special expert systems .Each employs a knowledge base,expressed in terms of relevant fuzzy inference rules, and an appropriate inference engine to solve this control problem which vary substantially according to the nature of the control problems in this research the control problem is a complex task in this robotics research which require a multitude of coordinated actions such a s maintaining a prescribed state of single variable, which are capable of utilizing knowledge elicited from human operators extended to dynamic robotic system with nonmonotone neural networks.
\end{abstract}

Keywords - Fuzzification, Fuzzy controllers, Expert System, Fuzzy sets, Quantization, Nonmonotone, Dynamic Robotic system

\section{Dr. Ashok Kumar Ramadoss \\ MCA, MBA, MSc Ms (fac. of engineering), PhD Humanoid Robotics, \\ Senior Member UACEE, New York, IRED \\ Professor. \\ Department of Computing, \\ Research in the Department of Mechanical engineering.}

Coimbatore Institute of Technology, (Affiliated to Anna University)

Coimbatore, India. Pincode 641014.

\section{INTRODUCTION}

In this research it has been observed that experienced results are able to perform and recognized that the areas of fuzzy systems and neural networks are strongly interconnected, Here in this research neural networks have been proven Fuzzification very useful in this Humanoid Robotic research using fuzzy set for constructing membership functions of relevant fuzzy sets and other context dependent entities from sample data. We had explored, Here the motivation for approximating fuzzy systems by neural networks is based upon the inherent capability of neural networks .This is relevant to fuzzy controllers and more for fuzzy expert systems that processed large numbers of fuzzy inference rules in this real time research. The concept of information is intimately connected with the concept of uncertainty. The most fundamental aspect of this connection is that uncertainty involved in any problem-solving situation is a result of some information deficiency , Information pertaining to the model within which the situation is conceptualized may be incomplete,imprecise,fragmentary, not fully reliable,vague ,contradictory, or deficient, In this research we measured the uncertainty involved in a problem solving situation in forced uncertainty by quantization in Nonmonotone conceptualized in a particular methodology in fuzzy controllers to extract structure and motion environments. This is crucial in control problems for which it is difficult or even impossible to construct precise mathematical models, or for which the acquired models are difficult or 
Proc. of the Fifth Intl. Conf. Advances in Computing, Communication and Information Technology- CCIT 2017

Copyright (C) Institute of Research Engineers and Doctors, USA .All rights reserved.

ISBN: 978-1-63248-131-3 doi: 10.15224/ 978-1-63248-131-3-10

expensive to use. These difficulties results from inherent nonlinearities.

\section{Problem definition}

In the above perspective the traditional techniques of system $[1,2,3,4,5]$ analysis are not well suited for dealing with humanistic systems because they fail to come to grips with the reality of the fuzziness of human thinking and behavior, [6]. Thus to deal with such systems radically, we need approaches which do not make a fetish of precision, rigor and mathematical formalism, and which employ instead a methodological framework [7], which is tolerant of imprecision and partial truths [8].

\section{Proposed Research}

The area of fuzzy systems has chosen in this research in the area of fuzzy control, it is thus appropriate to research in fuzzy control Fig (1). Our research in fuzzy control includes a connection between fuzzy controllers and neural networks; also we worked in the issue of fuzzifying neural networks from Dynamic Robotic System from the opted and forced uncertainty.

\subsubsection{Fuzzy Classifications}

Here the 8 linguistic states are distinguished for each variable, represented by triangular shape fuzzy numbers equally spread over the range of values the variable have the same range[-7,7] which is the range[-a,a]

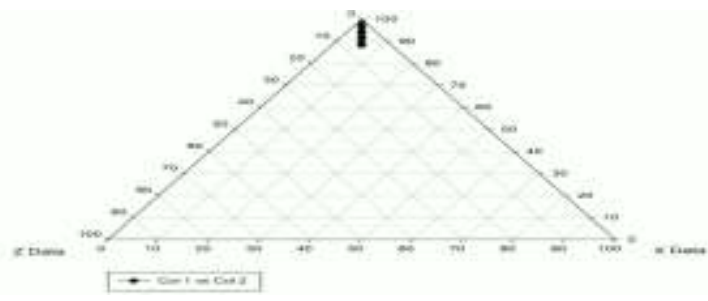

Fig 1. Fuzzy values plotted for various neural weights and neural schemas

\subsubsection{Fuzzy Algorithm}

In this research in the design of fuzzy controllers and the analysis of them by the below control logic are The relevant input and output variable of the Humanoid Robotic controller Fig (2) and ranges of their values selected linguistic states $[9,10,11]$ for each variable and express by appropriate fuzzy sets.

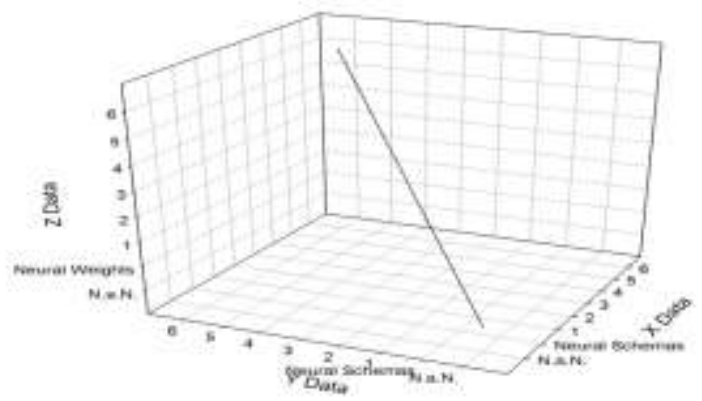

Fig 2. Fuzzy membership functions on Neural Schema with Neural Weight

A fuzzification function $f_{a}$ applied to a variable e. and made the fuzzification form $f_{a}:[-\mathrm{a}, \mathrm{a}] \rightarrow \mathrm{R}$ fig (2). Where $\mathrm{R}$ denotes the set of all fuzzy numbers and $f_{e}\left(x_{0}\right)$ is a fuzzy number chosen by $f_{e}$ as a fuzzy approximation of the measurement $\mathrm{e}=x_{0}$, the fuzzy set $f_{\theta}\left(x_{0}\right)$ enters into the inference process $[12,13]$. This control problem is formulated in terms of a set of fuzzy inference rules, here it has a canonical form [14.15]. If $\mathrm{e}=\mathrm{A}$ and $\dot{e}=\mathrm{B}$, then $\mathrm{v}=\mathrm{C}$ Fig (3) fuzzy sets representing the linguistic states of variables e, $\dot{e}, \mathrm{v}$, respectively and made reasonable to define a degree of relevance of the rule based by eqn $i_{1}\left[i_{2}\left(\mathrm{~A}\left(x_{k}\right), B\left(y_{k}\right)\right), C\left(z_{k}\right)\right]$, where $i_{1}, i_{2}$ are t-norms and the measurement of input.

\section{Fuzzyfying the Backpropagation learning algorithm in neurons}

In this research all real numbers that characterize a classical neural network become fuzzy numbers in its fuzzified 
Proc. of the Fifth Intl. Conf. Advances in Computing, Communication and Information Technology- CCIT 2017 Copyright (C) Institute of Research Engineers and Doctors, USA .All rights reserved.

ISBN: 978-1-63248-131-3 doi: 10.15224/ 978-1-63248-131-3-10

counterpart. Inputs to network and outputs of neural at hidden layers $\mathrm{O} O \mathrm{~N}_{k}$, of a neuron then the inputs $X_{K 0} X_{K 1}, \ldots, X_{K n}$, the weights $W_{0}, W_{1}, \ldots, W_{n}$, and the output $Y_{k}$ of this neuron are all fuzzy numbers. The output of each neuron, exemplified here by the neuron is given ineqn. $Y_{K}=S_{\beta}\left(\sum_{j=0}^{n} W_{j} X_{k j}\right)$, Where $S_{\beta}$ is a sigmoidal function for the chosen value of fig (3), the steepness parameters $\beta, W_{j} X_{k j}$ is the designate fuzzy number and the sum is $A_{k}=\sum_{j=0}^{n} W_{j} X_{k j}$ are calculated by fuzzy arithmetic and the output of the neuron obtained is determined by using the extension principle, Error function $E_{p}$, is employed in Fig 4.

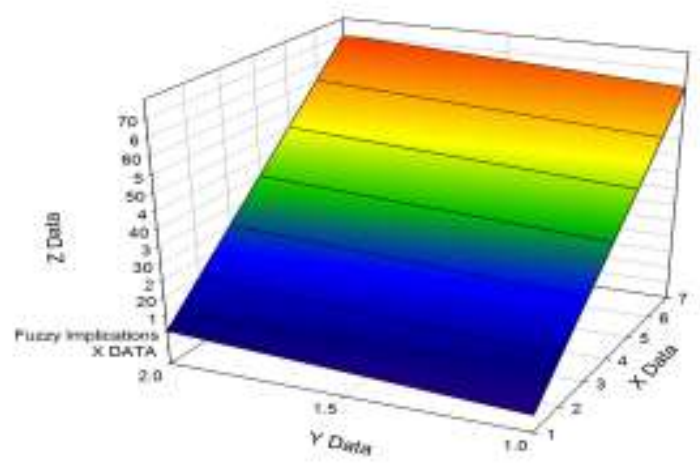

Fig. 3 Fuzzy implications

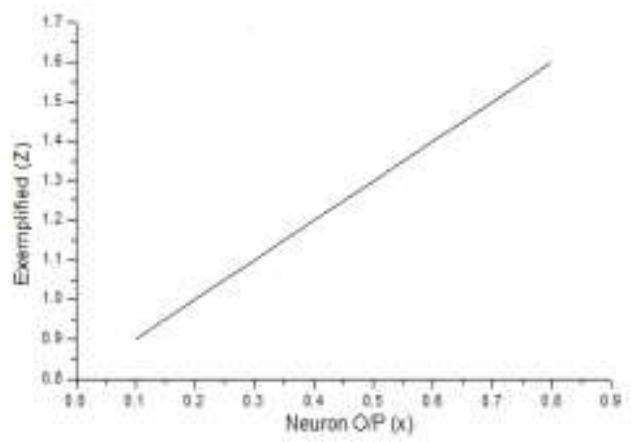

Fig.4 Nonmonotone Neuron Exemplified

Our back propagation learning algorithm $\mathrm{n}$ a fuzzy network with $m$ outputs for each training sample p Fig(5), is defined by the eqn.

$$
\begin{aligned}
& E_{p}=\frac{1}{2} \sum_{k}^{n}\left(T_{k}^{p}-Y_{k}^{p}\right)^{2} \\
& \lambda=\frac{1}{2} \sum_{k=1}^{n}\left(t_{k_{2}}^{p}-t_{k_{1}}^{p}\right)^{2}
\end{aligned}
$$

With their fuzzy counterparts and applying fuzzy arithmetic.

\section{Outcome - Fuzzifications of classical dynamic systems}

$\mathrm{U}$ is a set of time functions, $\mathrm{u}: \mathrm{T} \rightarrow \mathrm{X}$, which is required to be closed under a composition Fig (5) that is called a $\tau$ concatenation and implemented as follows: for any $u, v \in U$ and $\tau \in T$, the $\tau$ concatenation, $\mathrm{u} 0_{\tau} \mathrm{V}$, is the function fig $4 . \mathrm{S}$ is a global state-transition functions: $\mathrm{T} \times \mathrm{T} \mathrm{x}$ $\mathrm{Z} \times \mathrm{U} \rightarrow \mathrm{Z}$, which is required to have the following properties' $(\mathrm{t}, \mathrm{t}, \mathrm{z}, \mathrm{u})=\mathrm{Z}$ (consistency); $\mathrm{s}\left(t_{3}, t_{1}, \mathrm{z}, \mathrm{u}\right)=$ $\mathrm{s}\left[t_{3} t_{2}, s\left(t_{2}, t_{1}, \mathrm{z}, \mathrm{u}\right), \mathrm{u}\right]$ for any $t_{1} \leq t_{2} \leq t_{3}$ (state-substitution property); $\left(t_{2}, t_{1}, \mathrm{z}, \mathrm{u}\right)=\mathrm{s}\left[t_{2} t_{1}, \mathrm{z}, \mathrm{v}\right)$ when $\mathrm{u}(\mathrm{t})$ $=\mathrm{v}(\mathrm{t})$ for $t_{1} \leq t \leq t_{2}$ (causality); $\mathrm{r}$ is a response function used in $\mathrm{r}: \mathrm{T} \times \mathrm{Z} \times \mathrm{A} \rightarrow$ $\mathrm{B}$,. One way of fuzzifying these systems is done Fig (5), by using linguistic approximation from datas in the Neural Schema and Neural weights for different Humanoid Robot complexities.

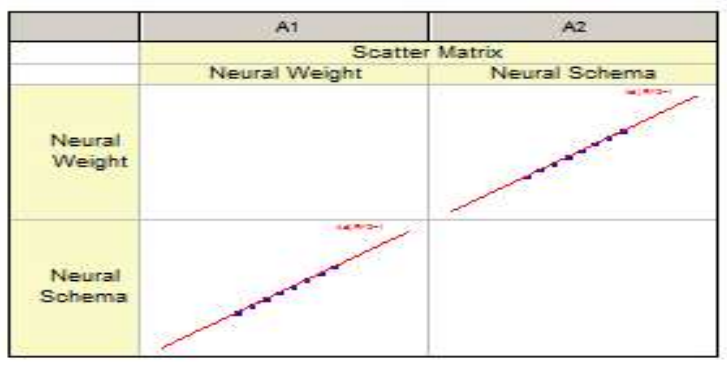

Fig. 5 Derived approximation as nonmonotone and Neural Weight V/S Neural Schema computational equivalence. 
Proc. of the Fifth Intl. Conf. Advances in Computing, Communication and Information Technology- CCIT 2017 Copyright (C) Institute of Research Engineers and Doctors, USA .All rights reserved.

ISBN: 978-1-63248-131-3 doi: 10.15224/ 978-1-63248-131-3-10

\section{Results and Discussions}

In this research the following are the results achieved in computational equivalence between continuous functions, neural networks, and fuzzy expert systems each neuron,provided that the range of input variable is discretized into $\mathrm{n}$ values and the range of the output variable is discretized into $\mathrm{m}$ values of Humanoid Robotic System Fuzzification.

\section{Experimental Validation for fuzzy inference}

Fuzzy input-output controllers, that is fuzzy expert systems based on multiconditional approximate reasoning and a defuzzification of obtained conclusions are universal approximates. Humanoid Robotic Neural networks with $\mathrm{n}$ inputs, $\mathrm{m}$ outputs( $(\mathrm{n} \geq 1, \mathrm{~m} \geq 1)$, one hidden layer and a continuous activation function in the sigmoid function in each neuron are universal approximates as given in the figures of humanoid robotic system in the experimental platform.

\section{Conclusion}

In this research in approximate linguistic terms is efficient, thus the role of uncertainty in reducing complexity is well characterized, an alternative approach is also developed on the premise that the key elements in human thinking are not numbers, but labels of fuzzy sets. Thus logic with fuzzy truths, Humanoid Robotic fuzzy connectives and fuzzy rules of inference is obtained for Dynamic Robotic System from the opted and forced uncertainty by quantization in Nonmonotone neural networks executes as gentle walk, walking backwards towards wall, sensing and trajectory towards right and left locomotion.

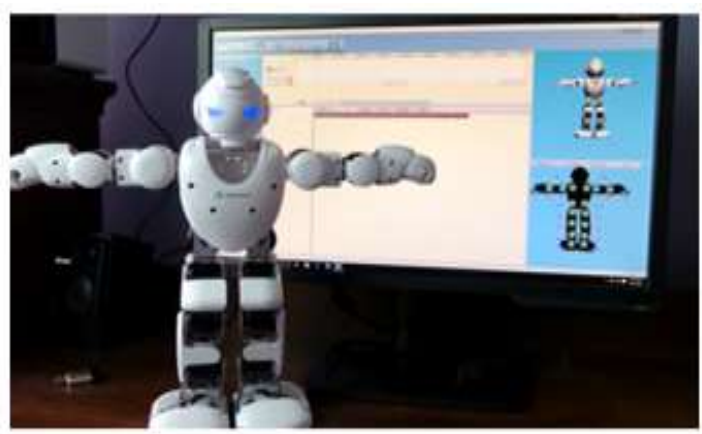

Comparisons of simulation Vs experimental platform (output experimental values are plotted as fuzzy values)

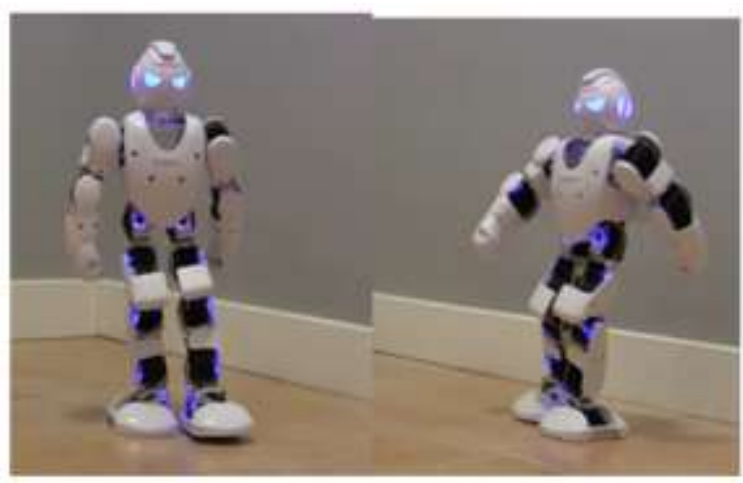

Gentle walk Walking backwards towards wall

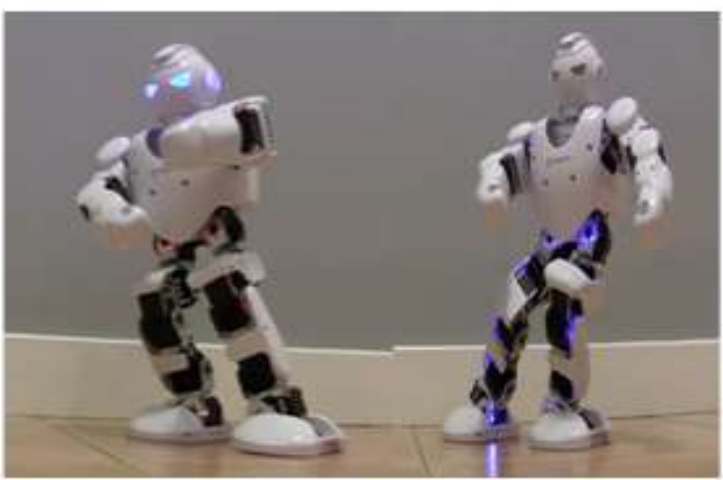

Walking torrards right

Walking towards left 
Proc. of the Fifth Intl. Conf. Advances in Computing, Communication and Information Technology- CCIT 2017

Copyright (C Institute of Research Engineers and Doctors, USA .All rights reserved.

ISBN: 978-1-63248-131-3 doi: 10.15224/ 978-1-63248-131-3-10

\section{Future work}

Another way is to apply the extension principle directly to the differential equations involved, this requires in some sense the development of a fuzzy differential calculus. Also can show how a proper defuzzification method can be determined by Humanoid Robotic learning algorithms, an interesting strategy for dealing with the defuzzification problem based on sensitivity analysis by applying the predictions.

\section{REFERENCES}

[1] "Fuzzy sets," Inf. Control, vol. 8, no. 3, pp. 338-353, 1965

[2] A. Saffiotti, "The uses of fuzzy logic in autonomous robot navigation," J. Soft Comput., vol. 1, no. 4, pp. 180-197, 1997.

[3] E. Tunstel, T. Lippincott, and M. Jamshidi, "Behavior hierarchy for autonomous mobile robots: Fuzzy behavior modulation and evolution," Int. J. Intell. Automat. Soft Comput., vol. 3, no. 1, pp. 37-49, 1997.

[4]Ashok Kumar Ramadoss , K. Marimuthu , Investigation on Data Concurrency for Sensory Fusion in Humanoid Robot, International Journal of Advancements in Mechanical and Aeronautical Engineering, Vol 1 , page(s):4549,May 2013.

[5] Uncertain Schema Matching Based on Fuzzy Decision Making (WENG Nian-feng,

DIAO Xing-chun and CAO Jian-jun) , INFORMATION - An International Interdisciplinary Journal, International Information Institute, Tokyo, Japan, Vol.15, No.11(B), pp.5125-5136, Novemver, 2012.

[6] Ashok Kumar Ramadoss , Marimuthu Krishnaswamy , Prediction of Stochastic Cognitive Neural Schema with Neural Network Paradigm of Latent Semantic Nodes in Autobot Humanoid Robot Using Nonlinear Regression of Gaussian Sigmoidal Curves in Boltzmann Normalisation INFORMATION - An International Interdisciplinary Journal, International Information Institute, Tokyo, Japan. Vol.17, No.8, pp.3971-3984, 2014.

[7] Mobile Robot Active Localization using a Learned Bayesian Network ,Hongjun Zhou and
Zhuping Wang, INFORMATION - An International Interdisciplinary Journal, International Information Institute, Tokyo, Japan, Vol.17, No.2, pp.747-776, February, 2014.

[8]. Johannsen G (1972) Development and optimization of a non linear multiparameter human operator model. IEEE Tr. Sys. Man, Cyber. 2(4): 494-504.

[9]. Costello R G (1968) The surge model of the well - trained human operator in simple manual control. IEEE Tr. Man-Mach. Sys. MMS - 9(1): 2-9.

[10]. A. Zhu, S.X. Yang, Neuro fuzzy based approach to mobile robot navigation in unknown environments, IEEE Trans. Syst. Man Cybern. C 37 (2007) 610-621.

[11]. W.L. Xu, S.K. Tso, Sensor-based fuzzy reactive navigation of a mobile robot through local target switching, IEEE Trans. Syst. Man Cybern. C 29 (1999) 451-459.

[12].Carpenter G A, Grossberg S, Markuson N, Reynolds J H, Rosen D B (1992) Fuzzy ARTMAP:A neural network architecture for incremental supervised learning of analog multidimensional maps. IEEE Trans. on Neural Networks 3(5): 698-713.

[13] Ashok Kumar Ramadoss , Marimuthu Krishnaswamy, Embedding inference engine in fuzzy expert robotic system shell in a humanoid robot platform for selecting stochastic appropriate fuzzy implications for approximate reasoning, DOI 10.1007/s10015-014-0189-2 Artif Life Robotics (2015) 20:13-18,Springer Germany.

[14]. R. Ivey, D. Bullock, S. Grossberg, A neuromorphic model look ahead planning, Neural Networks 24 (3) (2011) 257-266.

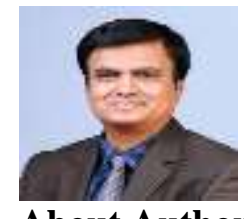

About Author

$\mathrm{He}$ is the Best Paper Award Winner at KL Malaysia and chaired the session in Robotics International Conference. $\mathrm{He}$ is the Senior Global Member UACEE IRED, New York, USA and a Humanoid Robotics Scientist. 